\title{
Suppression of cyclooxygenase 2 increases chemosensitivity to sesamin through the Akt-PI3K signaling pathway in lung cancer cells
}

\author{
QING FANG $^{1}$, YUYIN ZHU ${ }^{1}$, QILAI WANG ${ }^{1}$, MEIJUN SONG ${ }^{2}$, GUOSHENG GAO ${ }^{3}$ and ZHIMING ZHOU ${ }^{1}$ \\ Departments of ${ }^{1}$ Pulmonary Medicine, ${ }^{2}$ Emergency Medicine and ${ }^{3}$ Laboratory, Ningbo No. 2 Hospital, \\ Ningbo, Zhejiang 315000, P.R. China
}

Received June 12, 2018; Accepted October 1, 2018

DOI: 10.3892/ijmm.2018.3939

\begin{abstract}
Safe, affordable and efficacious agents are urgently required for cancer prevention. Sesamin, a lipid-soluble lignan from sesame (Sesamum indicum) displays anticancer activities through an unknown mechanism. In the present study, the anticancer activity of sesamin via cyclooxygenase 2 (COX2) was investigated in lung cancer. Quantitative polymerase chain reaction was performed to determine the mRNA expression levels of COX2 in cells, while western blot analysis was used to determine its protein expression levels. Cell proliferation was evaluated by Cell Counting Kit- 8 assay, while apoptosis and cell cycle analyses were conducted by flow cytometry. The results indicated that $\mathrm{COX} 2$ expression was upregulated in lung cancer cell lines compared with human normal lung epithelial cell line BEAS-2B and sesamin was demonstrated to decrease the levels of COX2, inhibit the proliferation of lung cancer cells and promote their apoptosis in a concentration-dependent manner. Furthermore, decreased COX2 expression potentiated sesamin-induced apoptosis and G1-phase arrest, which was correlated with the suppression of gene products associated with cell apoptosis (Bcl-2 and Bax) and the cell cycle (cyclin E1). In addition, cotreatment with the COX2 inhibitor CAY10404 and sesamin downregulated the expression of downstream molecules of COX2 [including interleukin (IL) $1 \beta$, IL6 and tumor necrosis factor $\alpha$ ] compared with CAY10404 or sesamin alone. Furthermore, cotreatment with sesamin and CAY10404 markedly reduced the levels of phosphorylated protein kinase B (pAkt) and phosoinositide 3 kinase (PI3K) in three lung cancer cell lines. PI3K expression was observed to be under the control of $\mathrm{COX} 2$, possibly forming a negative
\end{abstract}

Correspondence to: Professor Qilai Wang, Department of Pulmonary Medicine, Ningbo No. 2 Hospital, 41 Xibei Street, Ningbo, Zhejiang 315100, P.R. China

E-mail: 394901528@qq.com

Key words: lung cancer cells, sesamin, cyclooxygenase 2, protein kinase B-phosphoinositide 3 kinase signaling pathway, chemosensitivity feedback loop. In addition, PI3K depletion induced apoptosis and G1-phase arrest in A549 cells. These results suggested that sesamin blocked the pAkt-PI3K signaling pathway by downregulating the expression of $\mathrm{COX} 2$, therefore resulting in cell cycle arrest and increased apoptosis in vitro. In conclusion, inhibition of COX2 increased the sensitivity of lung cancer cells to sesamin by modulating pAkt-PI3K signaling. These results may aid the development of more selective agents to overcome cancer.

\section{Introduction}

Lung cancer is the most common malignancy and the leading cause of cancer-associated mortality worldwide (1). In the USA, there were 222,500 newly diagnosed cases of lung cancer and 155,870 mortalities due to lung cancer in 2017, according to statistics reported by Siegel et al (2). Currently, there are two recognized subtypes of lung cancer, non-small cell lung cancer (NSCLC) and small cell lung cancer. Clinically, NSCLC accounts for $>80 \%$ of the total incidence of lung cancer (3). Chemotherapy is one of the most effective solutions for lung cancer. However, due to the frequent alterations in chemotherapy regimens, chemotherapy resistance is a major problem in clinical practice and, importantly, its underlying mechanisms remain to be elucidated (4).

Sesamin, a type of lignan, is a biologically active compound extracted in large quantities from Sesamum indicum (5). Sesamin has various valuable biological functions, including protection against oxidative stress, anti-inflammation and the inhibition of carcinogenesis (6). A previous study revealed that sesamin decreased the frequency of chemical induction of breast tumors and enhanced liver detoxification (5). In addition, sesamin reduced lipopolysaccharide (LPS)-induced acute lung injury in mice (7) and inhibited LPS-induced cell proliferation and invasion in prostate cancer cells (8). However, the effect of sesamin on lung cancer and the molecular mechanism implicated in this process remain largely unknown.

Cyclooxygenase 2 (COX2) is one of the two subtypes of COX, which is considered to be involved in inflammation and carcinoma progression (9). COX2 may be induced by various stimuli, including growth factors, oncogenes, cytokines and 
hormones (10). It has been reported that COX2 may serve an important role in lung carcinogenesis. Overexpression of COX2 is common in NSCLC and appears to be associated with tumor progression and metastasis (11). However, whether COX2 regulates chemosensitivity toward sesamin in lung cancer cells and the mechanisms associated with this process, are still under investigation.

The present study investigated the effect of COX2 on sesamin-induced apoptosis and the cell cycle in lung cancer cells via the Akt/PI3K signaling pathways. The present results may provide a valuable insight into the mechanism of sesamin-induced progression and development of lung cancer.

\section{Materials and methods}

Cell culture. The lung cancer cell lines A549, NCI-H446 and H1299 were purchased from the American Type Culture Collection (Manassas, VA, USA). The human normal lung epithelial cell line BEAS-2B was obtained from the Stem Cell Bank of the Chinese Academy of Sciences (Shanghai, China). All cell lines were cultured in RPMI-1640 medium (HyClone; GE Healthcare Life Sciences, Logan, UT, USA) containing $10 \%$ fetal bovine serum (ExCell Bio, Inc., Shanghai, China) and incubated at $37^{\circ} \mathrm{C}$ with $5 \% \mathrm{CO}_{2}$. Lung cancer cells were treated with $25 \mu \mathrm{M}$ CAY10404 (a COX2 inhibitor; Santa Cruz Biotechnology, Inc., Dallas, TX, USA) for $2 \mathrm{~h}$. Subsequently, the cells were incubated for additional $24 \mathrm{~h}$ with $50 \mu \mathrm{M}$ sesamin (Beijing Solarbio Science \& Technology Co., Ltd., Beijing, China). In the case of A549 cells, these were treated with $10 \mu \mathrm{M}$ LY294002 (a PI3K inhibitor; Thermo Fisher Scientific, Inc., Waltham, MA, USA) for $24 \mathrm{~h}$ (12).

Cell Counting Kit (CCK)-8 assay. Cell proliferation was detected by CCK-8 analysis (Dojindo Molecular Technologies, Inc., Rockville, MD, USA). Briefly, lung cancer cells $\left(5 \times 10^{3}\right.$ cells $/ 100 \mu \mathrm{l} /$ well $)$ were seeded in a 96 -well plate (Corning Incorporated, Corning, NY, USA). After $24 \mathrm{~h}$, the cells were treated with $0,10,50,100$ and $150 \mu \mathrm{M}$ sesamin for

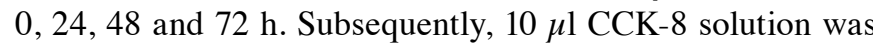
added to each well and the absorbance at $450 \mathrm{~nm}$ was read following incubation for $3 \mathrm{~h}$ at $37^{\circ} \mathrm{C}$ (3).

Cell apoptosis assay. Cell apoptosis was analyzed using an apoptosis detection kit [Multisciences (Lianke) Biotech Co., Ltd., Hangzhou, China] according to the manufacturer's protocol. In brief, treated lung cancer cells were resuspended in $500 \mu 1 \mathrm{X}$ Binding Buffer and incubated with $10 \mu \mathrm{l}$ propidium iodide (PI) and $5 \mu \mathrm{l}$ Annexin V-fluorescein isothiocyanate (FITC). Following 5 min of incubation in the dark, flow cytometric analysis was conducted using a FACScan flow cytometer (BD Biosciences, Franklin Lakes, NJ, USA) and a FlowJo 7.6 software (1997-2008; FlowJo LLC, Ashland, OR, USA) (13).

Cell cycle assay. Cell cycle was determined with a cell cycle detection kit [Multisciences (Lianke) Biotech Co., Ltd.] according to the manufacturer's protocol. In brief, treated lung cancer cells were resuspended in $10 \mu \mathrm{l}$ permeabilization solution and $1 \mathrm{ml}$ DNA staining solution. Following 30 min of staining at room temperature, flow cytometric analysis was conducted using a FACScan flow cytometer (BD Biosciences) (14).

Western blot assay. The cells were lysed in radioimmunoprecipitation assay buffer (Beijing Solarbio Science \& Technology Co., Ltd., Beijing, China). Protein concentration was determined with a bicinchoninic acid protein assay kit (Beyotime Institute of Biotechnology, Haimen, China). Proteins (40 $\mu \mathrm{g} / \mathrm{sample}$ ) were separated by $12 \%$ SDS-PAGE and transferred to a polyvinylidene fluoride membrane $(0.2 \mu \mathrm{M}$; EMD Millipore, Billerica, MA, USA). The membranes were blocked in $5 \%$ bovine serum albumin (Beijing Solarbio Science \& Technology Co., Ltd.) in TBS with $0.1 \%$ Tween-20 for $2 \mathrm{~h}$ at room temperature and incubated with primary antibodies at $4^{\circ} \mathrm{C}$ overnight. Antibodies against COX2 (cat. no. 12282), Bcl-2 (cat. no. 15071), Bax (cat. no. 5023), cyclin D1 (cat. no. 2922), cyclin B1 (cat. no. 12231), cyclin A2 (cat. no. 4656), interleukin (IL) $1 \beta$ (cat. no. 12242), IL6 (cat. no. 12153), tumor necrosis factor (TNF) $\alpha$ (cat. no. 3707), AKT (cat. no. 9272), pAKT (cat. no. 4060), PI3K (cat. no. 4257), mammalian target of rapamycin (mTOR; cat. no. 2972) and GAPDH (cat. no. 5174; 1:1,000; Cell Signaling Technology, Inc., Danvers, MA, USA) were used. Upon washing, the membranes were incubated with secondary antibodies [Goat anti-rabbit immunoglobulin (Ig)G-horseradish peroxidase (HRP) cat. no. BA1054 or goat anti-mouse IgG-HRP cat. no. BA1050; 1:5,000; Wuhan Boster Biological Technology, Ltd., Wuhan, China] for $2 \mathrm{~h}$ at room temperature. The protein bands were detected by chemiluminescence (Western blotting detection kit, cat. no. K-12045-D10; Advansta, San Jose, CA, USA). Densitometric analysis was performed by Tanon GIS version 4.1.2 software (Tanon Science and Technology Co., Ltd., Shanghai, China) (15).

\section{Reverse transcription-quantitative polymerase chain reaction} (RT-qPCR) assay. Total RNA was extracted from lung cancer cells using TRIzol ${ }^{\circledR}$ reagent (Invitrogen; Thermo Fisher Scientific, Inc.). The concentration of RNA was quantified using NanoDrop 2000 (NanoDrop Technologies; Thermo Fisher Scientific, Inc., Wilmington, DE, USA) and the RNA was then reverse-transcribed into cDNA using an RT kit (Thermo Fisher Scientific, Inc.). The procedure for reverse transcription included an annealing step of $10 \mathrm{~min}$ at $65^{\circ} \mathrm{C}$, followed $1 \mathrm{~h}$ at $42^{\circ} \mathrm{C}, 5 \mathrm{~min}$ at $85^{\circ} \mathrm{C}$ and immediately placed in ice. RT-qPCR was performed using LightCycler 480 SYBR-Green I Master (16) (Roche Applied Science, Madison, WI, USA) to detect COX2 mRNA expression. GAPDH was used as the internal control. The primer sequences used in RT-qPCR were as follows: COX2, 5'-TCAAAACCGAGGTGTA-3' (sense) and 5'-GTGGGTAAGTATGTAGTGC-3' (antisense); and GAPDH, 5'-AAGCCTGCCGGTGACTAAC-3' (sense) and 5'-GCATCACCCGGAGGAGAAAT-3' (antisense). The procedure for qPCR included an initial denaturation of $15 \mathrm{~min}$ at $95^{\circ} \mathrm{C}$, followed by 40 cycles of $10 \mathrm{sec}$ at $95^{\circ} \mathrm{C}, 22 \mathrm{sec}$ at $55^{\circ} \mathrm{C}$ and $30 \mathrm{sec}$ at $72^{\circ} \mathrm{C}(17)$.

Statistical analysis. All experiments were repeated three times. One-way analysis of variance and Fisher's exact test was performed to evaluate the differences between groups using SPSS 18.0 (SPSS, Inc., Chicago, IL, USA). The data are expressed as the mean \pm standard deviation. 
A

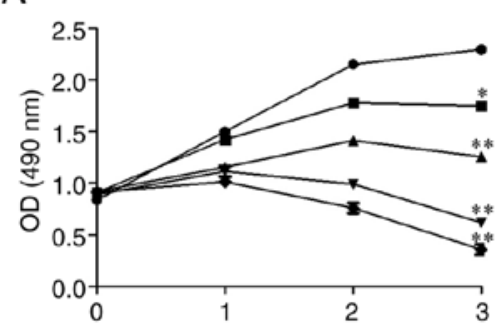

$\mathrm{NCl}-\mathrm{H} 446$

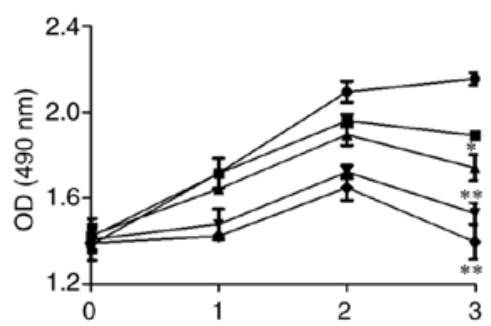

$\mathrm{H} 1299$

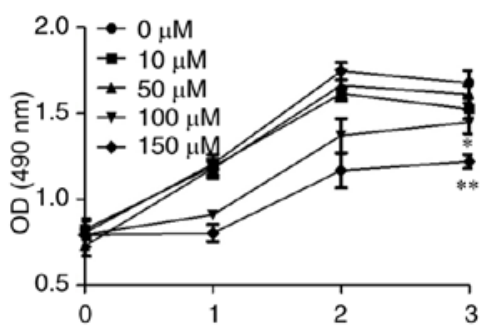

B
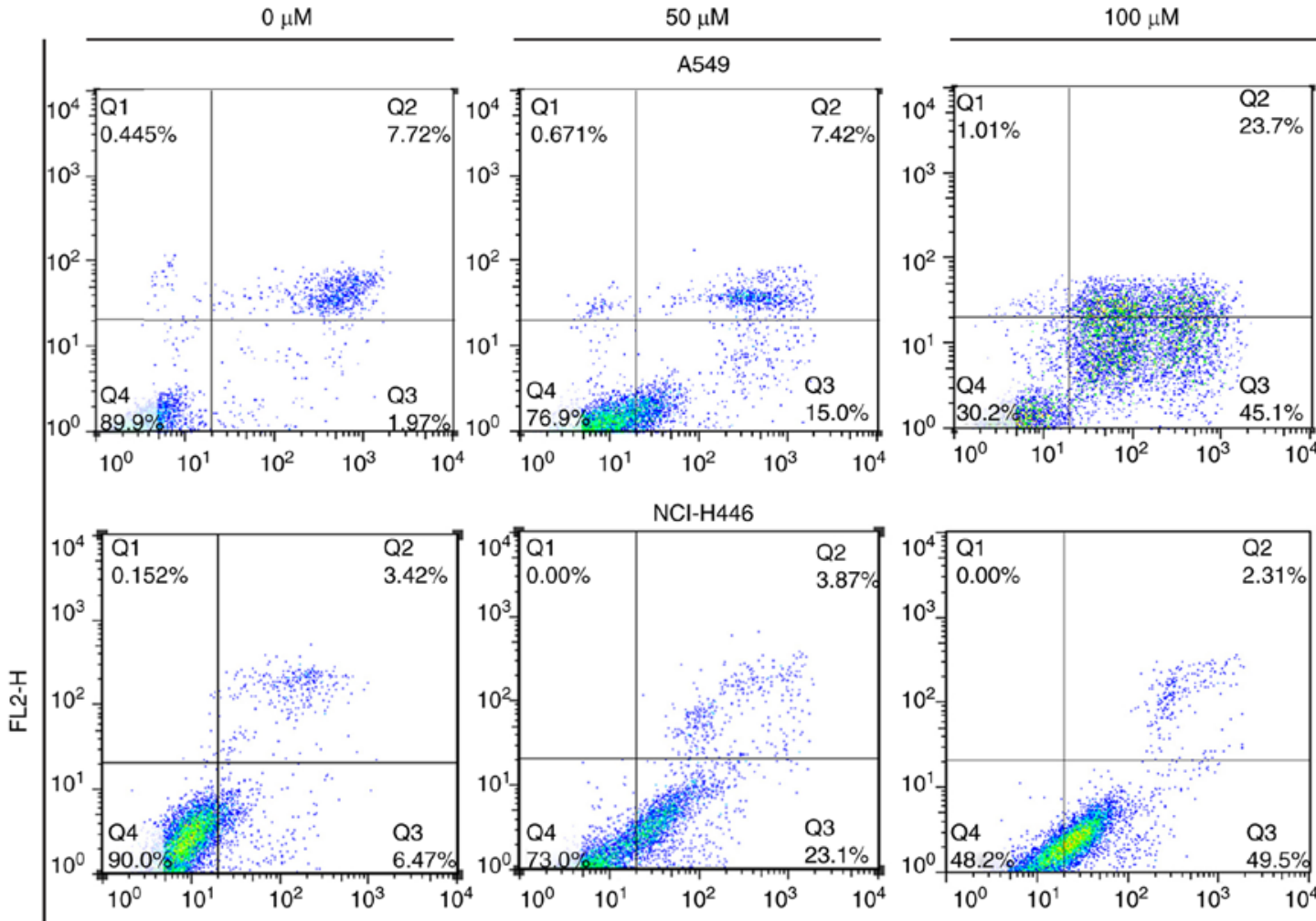

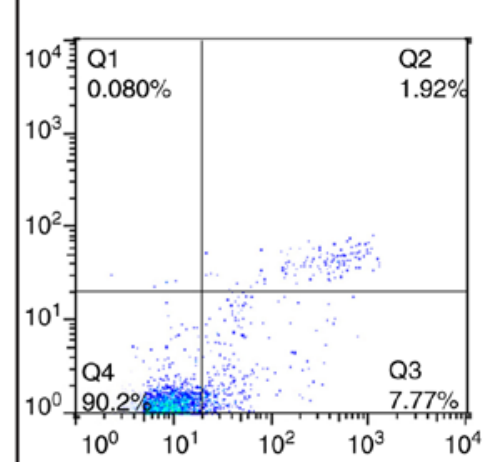

H1299
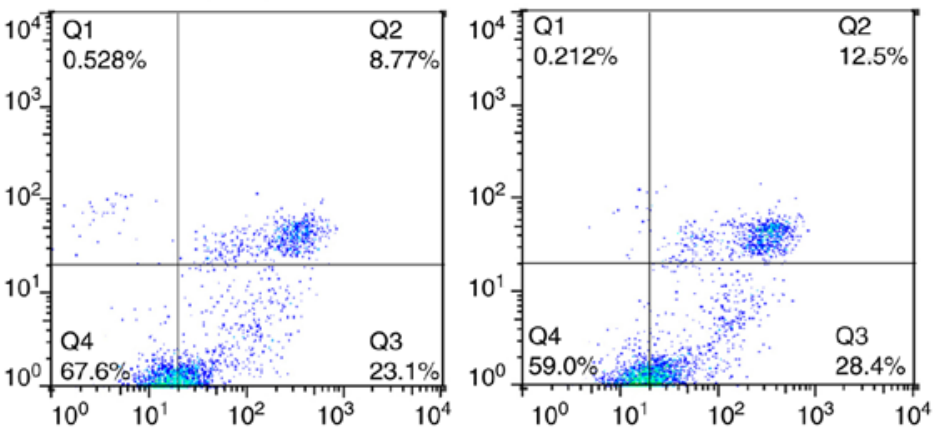

FL1-H

Figure 1. Sesamin inhibits the proliferation and promotes the apoptosis of lung cancer cells. (A) A549, NCI-H446 and H1299 cells were treated with 0, 10, 50, 100 and $150 \mu \mathrm{M}$ sesamin for 0,24, 48 and $72 \mathrm{~h}$, and the cytotoxicity of sesamin was detected by Cell Counting Kit- 8 assay. (B) The above three cell lines were treated with the indicated concentrations of sesamin for $24 \mathrm{~h}$ and flow cytometry analysis was performed to examine sesamin-induced apoptosis. "P<0.05 and ${ }^{* *} \mathrm{P}<0.01$ vs. $0 \mu \mathrm{M}$. p-Akt, phosphorylated protein kinase B, PI3K, phosphoinositide 3 kinase; mTOR, mammalian target of rapamycin; COX2, cyclooxygenase 2 .

$\mathrm{P}<0.05$ was considered to indicate a statistically significant difference.

\section{Results}

Cytotoxic effect of sesamin against human lung cancer cells. To evaluate the effect of sesamin on lung cancer cells,
A549, NCI-H446 and H1299 cells were treated with 0, 10, 50, 100 and $150 \mu \mathrm{M}$ sesamin for $0,24,48$ and $72 \mathrm{~h}$. Sesamin significantly inhibited the proliferation of lung cancer cells A549 and NCI-H446 in a concentration-dependent manner compared with the cells treated with $0 \mu \mathrm{M}$ sesamin, which served as the control ( $\mathrm{P}<0.05$; Fig. 1A). In H1299 inhibition was only significant at $100 \mu \mathrm{M}$ or over. Furthermore, the 
A

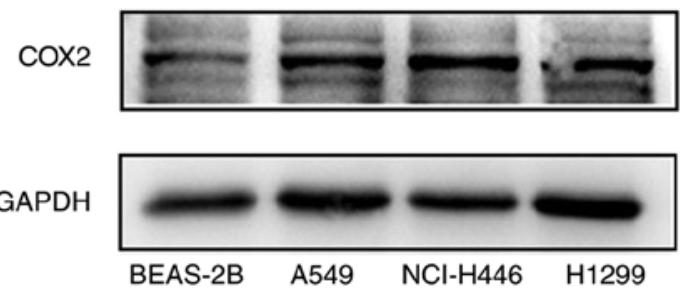

B

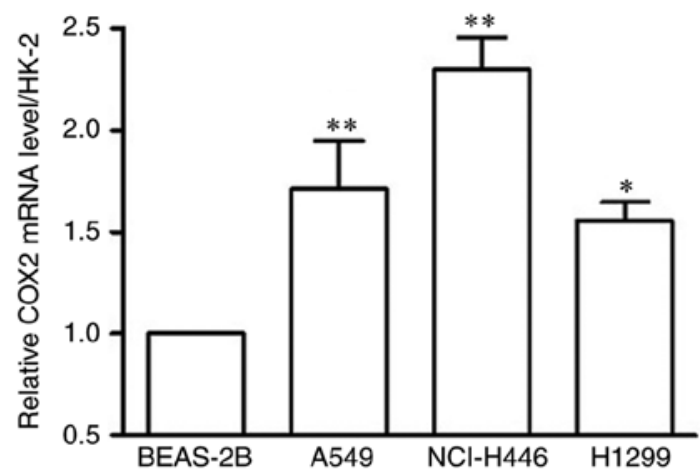

C

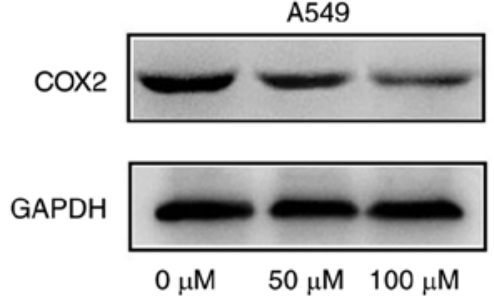

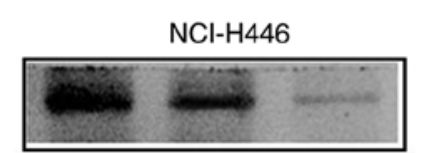

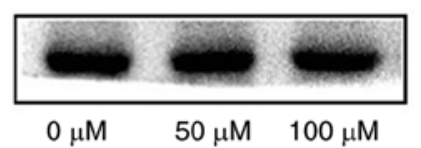

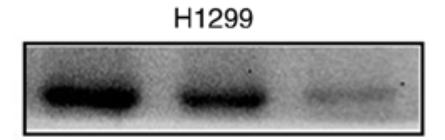

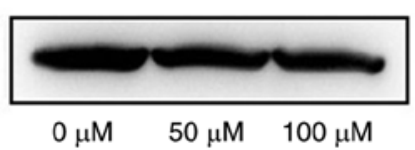

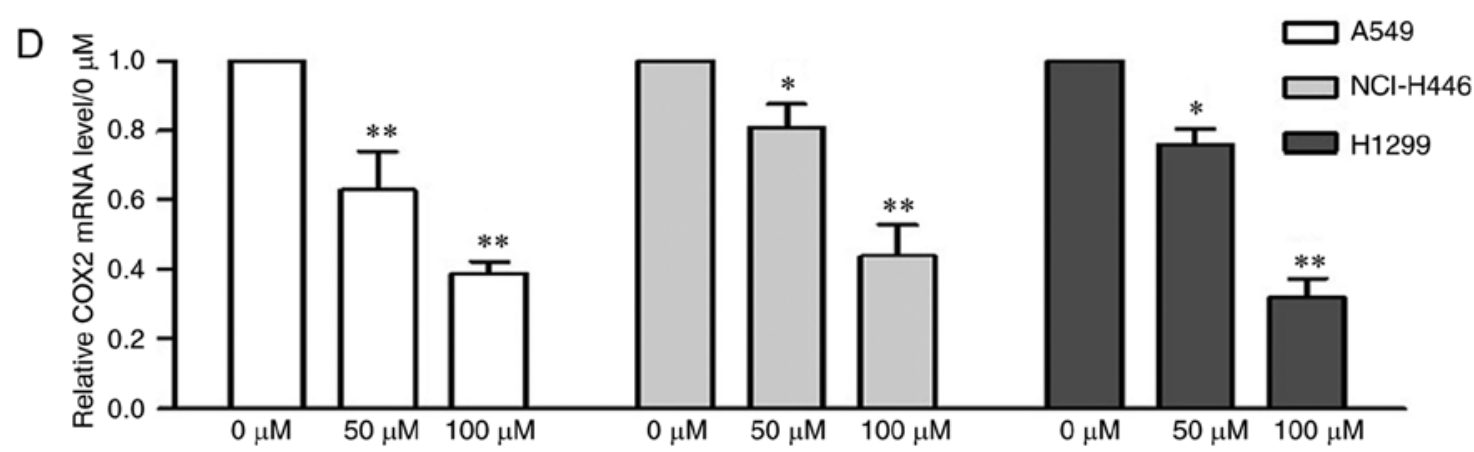

Figure 2. Sesamin downregulates the expression of COX2 in lung cancer cells. The protein and mRNA expression levels of COX2 were respectively evaluated by (A) western blotting and (B) quantitative polymerase chain reaction in three lung cancer cell lines and in BEAS-2B cells. " $P<0.05$ and ${ }^{* * *} \mathrm{P}<0.01$ vs. BEAS-2B cells. The lung cancer cells were treated with the indicated concentrations of sesamin for $24 \mathrm{~h}$. Subsequently, the (C) protein and (D) mRNA levels of COX2 were respectively analyzed as in (A) and (B). ${ }^{*} \mathrm{P}<0.05$ and $^{* *} \mathrm{P}<0.01$ vs. $0 \mu \mathrm{M}$. COX2, cyclooxygenase 2.

results of the flow cytometry analysis of Annexin V-FITC and PI double staining indicated that sesamin promoted the apoptosis of lung cancer cells in a concentration-dependent manner compared with the control (Fig. 1B). Therefore, these results suggested that sesamin possesses an antitumor role in lung cancer cells.

Sesamin regulates the expression of COX2 in lung cancer cells. To investigate the role of COX2 in the cytotoxic effect of sesamin on A549, NCI-H446 and H1299 cells, the expression of COX2 was detected in the above lung cancer cell lines. As presented in Fig. 2A and B, the protein and mRNA expression levels of COX2 were markedly upregulated in lung cancer cells compared with BEAS-2B cells. Furthermore, as presented in Fig. $2 \mathrm{C}$ and D, sesamin decreased the protein and significantly decreased the mRNA expression levels of COX2 in A549, NCI-H446 and H1299 cells in a dose-dependent manner $(\mathrm{P}<0.05)$. These results suggested that sesamin serves an antitumor role by regulating the levels of COX2 in lung cancer cells.

Inhibition of COX2 expression enhances sesamin-induced lung cancer cells apoptosis. To clarify the role of COX2 expression inhibition in sesamin-induced insensitivity of lung cancer cells, CAY10404, a COX2 inhibitor, was used. As presented in Fig. 3A and B, CAY10404 repressed the mRNA and protein level of COX2 compared with the control. In addition, lung cancer cells were treated with sesamin in the presence or absence of CAY10404. As presented in Fig. 3C and D, cotreatment with CAY10404 and sesamin significantly increased the apoptosis of lung cancer cells $(\mathrm{P}<0.01)$ and the expression of the pro-apoptotic protein Bax, whereas it decreased the expression of the anti-apoptotic protein Bcl-2. These results suggested that selective inhibition of COX2 enables sesamin to induce apoptosis in lung cancer cells.

Inhibition of COX2 expression enhances sesamin-induced cell cycle arrest. The present study determined that COX2 expression served a role in sesamin-induced cell cycle arrest in lung cancer cells. The percentage of G1-phase cells increased upon cotreatment with sesamin and CAY10404 (Fig. 4A). In addition, cotreatment with CAY10404 and sesamin significantly repressed expression of the G1-phase protein cyclin D1 $(\mathrm{P}<0.05)$ and the G2/M-phase protein cyclin B1, but stimulated expression of the S-phase protein cyclin A2 


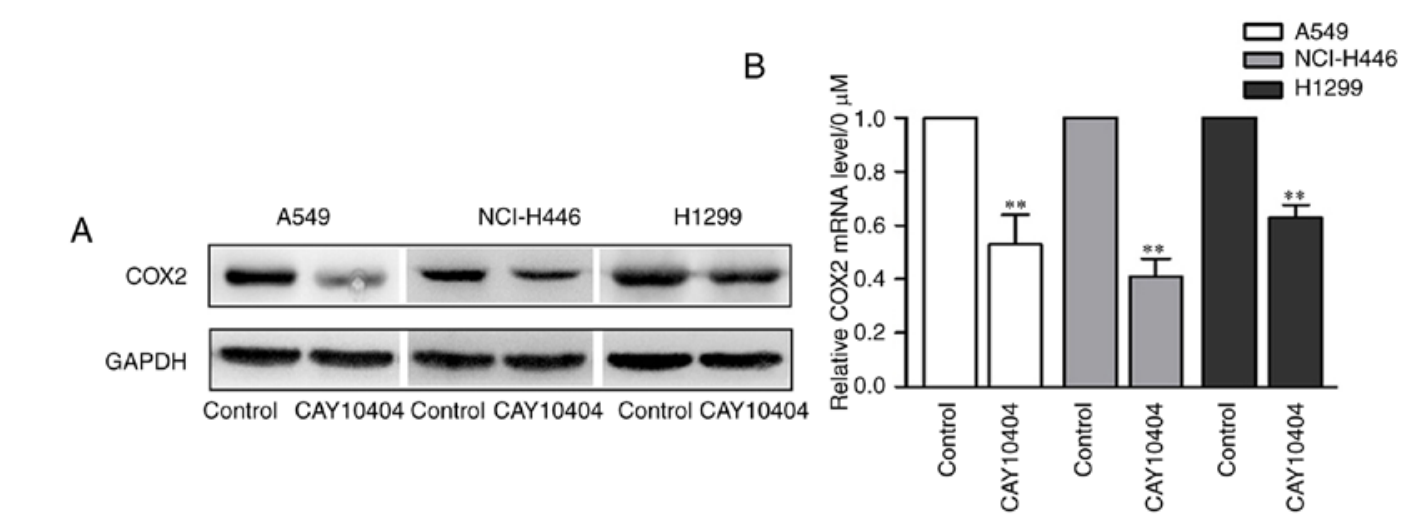

C
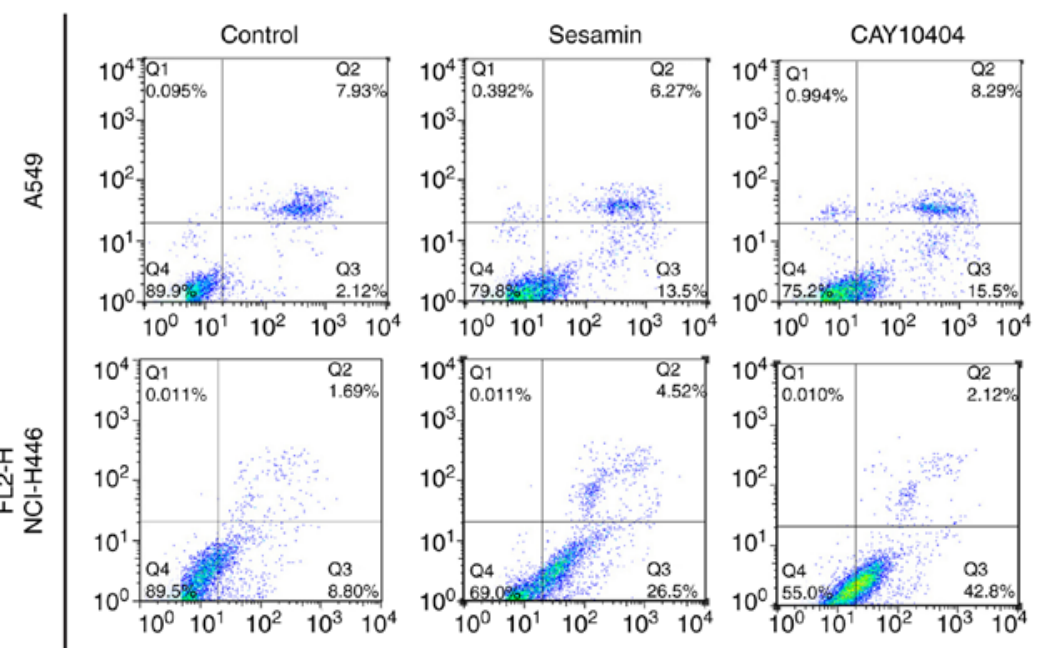

Sesamin+CAY10404
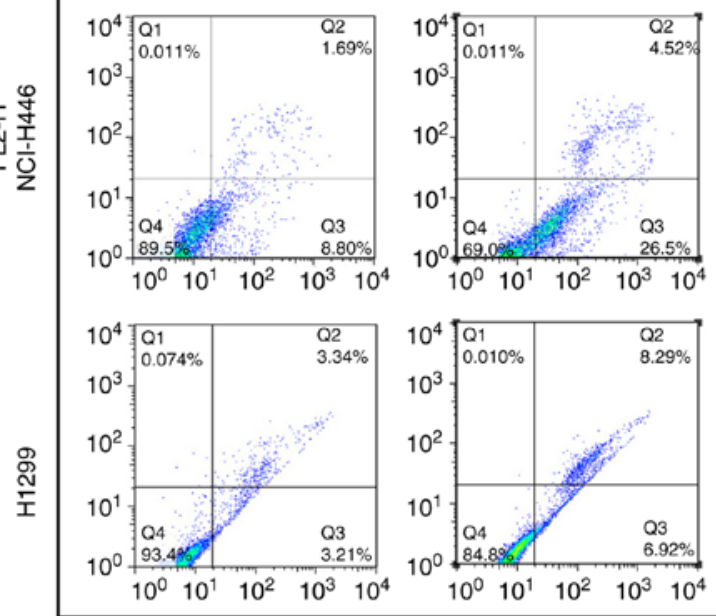

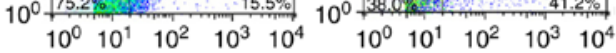
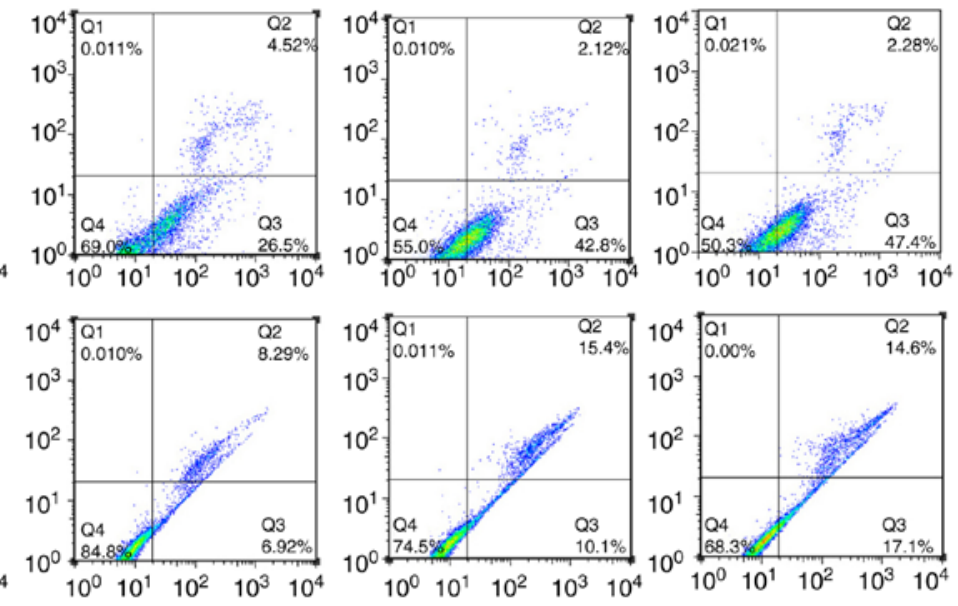

FL1-H

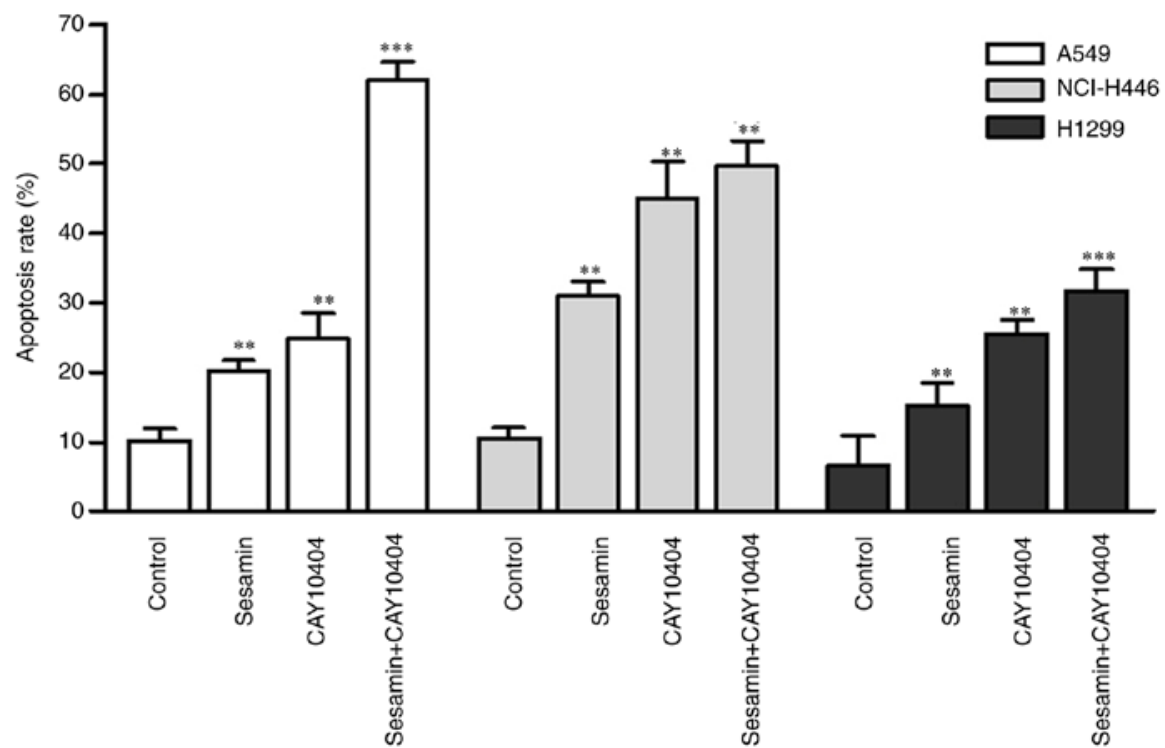

Figure 3. COX2 inhibition promotes sesamin-induced lung cancer cell apoptosis. Following treatment with $25 \mu \mathrm{M}$ CAY10404 (a COX2 inhibitor) for $24 \mathrm{~h}$, the protein and mRNA expression levels of COX2 were, respectively, evaluated by (A) western blotting and (B) quantitative polymerase chain reaction in A549, NCI-H446 and H1299 cells. (C) A549, NCI-H446 and H1299 cells were treated with $25 \mu \mathrm{M}$ CAY10404 for $2 \mathrm{~h}$ and incubated for additional $24 \mathrm{~h}$ with $50 \mu \mathrm{M}$ sesamin. Cell apoptosis was analyzed by flow cytometry upon staining with Annexin V-fluorescein isothiocyanate/propidium iodide. ${ }^{* *} \mathrm{P}<0.01$ and ${ }^{* * * *} \mathrm{P}<0.001$ vs. the control. COX2, cyclooxygenase 2 . 

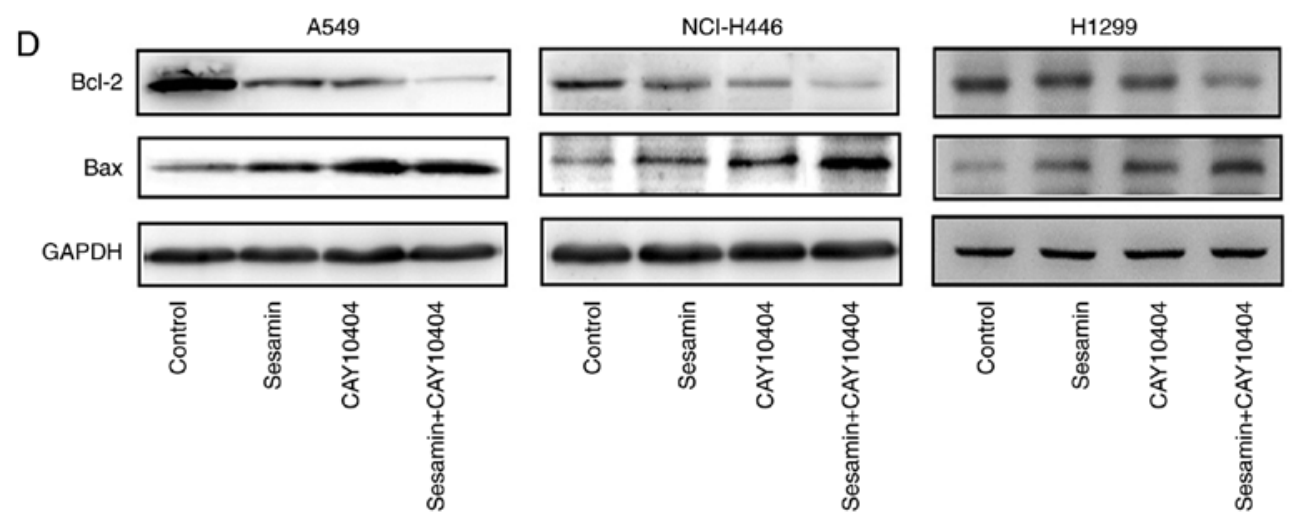

Figure 3. Continued. COX2 inhibition promotes sesamin-induced lung cancer cell apoptosis. (D) Cells were treated as in (C) and the expression of the pro-apoptotic protein Bax and the anti-apoptotic protein Bcl-2 was evaluated using western blotting.

(Fig. 4B). These results indicated that downregulated COX2 expression increased sesamin-induced G1-phase arrest, which was associated with the altered expression of cell cycle associated proteins.

Cotreatment with CAY10404 and sesamin strengthens the inhibition of the expression of COX2 downstream molecules. IL1 $\beta$, IL6 and TNF $\alpha$ are downstream molecules of COX2 (18). To further elucidate the involvement of COX2 in the sesamin-mediated regulation of lung cancer cells, the expression of the above downstream proteins was detected. The results indicated that the levels of these molecules were downregulated, particularly upon cotreatment with CAY10404 and sesamin (Fig. 5). These results suggested that sesamin exhibits an anticancer role in lung cancer cells and that downregulation of COX2 expression potentiates its antitumor effect.

Sesamin inhibits the pAkt-PI3K signaling pathway by inhibition of COX2 expression. To investigate the molecular mechanism of sesamin-induced apoptosis via COX2, the expression levels of Akt, pAkt, PI3K and mTOR in lung cancer cells treated with sesamin and/or CAY10404 were determined by western blotting. As presented in Fig. 6A, cotreatment with sesamin and CAY10404 markedly reduced the levels of pAkt and PI3K in three lung cancer cell lines. The PI3K inhibitor LY294002 decreased the expression of PI3K while partially upregulating the expression of COX2. However, the effect was not statistically significant in A549 cells (Fig. 6B). These results indicated that PI3K was under the control of COX2 and possibly formed a negative feedback loop. In addition, inhibition of PI3K expression induced apoptosis (Fig. 6C) and G1-phase arrest (Fig. 6D) in A549 cells. These results suggested that sesamin inhibits the pAkt-PI3K signaling pathway by decreasing the expression of COX2, which leads to cell cycle arrest and the induction of apoptosis in vitro.

\section{Discussion}

Lung cancer is the leading cause of cancer-associated mortality in males and females worldwide (19). Plant-derived agents are widely applied as adjuvant or supplemental agents in cancer therapy. Sesamin has gained attention recently due to its antitumor effects (20). The present study identified that sesamin is able to suppress the proliferation and promote the apoptosis of lung cancer cells in a concentration-dependent manner, indicating that sesamin is relatively effective in lung cancer, similar to the effects reported in other cancer types, including breast cancer (21), human hepatocellular carcinoma (22), colon cancer, prostate cancer and pancreatic cancer (23). Previous studies have demonstrated that COX2 expression is strongly associated with cancer progression in various human tumor types $(10,24)$. COX2 been reported to be one of the important target molecules in tumor treatment (25). Shimizu et al (26) observed that COX2 transcriptional activities decreased by $50 \%$ in the presence of $100 \mu \mathrm{M}$ sesamol (one of the lignans in sesame seeds), while other compounds in sesame seeds, including sesamin, did not exhibit significant inhibition of COX2 transcriptional activity at $\leq 100 \mu \mathrm{M}$ in colon cancer cells. However, the present study noted that COX2 was highly expressed in lung cancer cells and that sesamin dose-dependently decreased the protein and mRNA levels of COX2. Therefore, it is reasonable to consider that sesamin inhibits lung cancer development at least partially by decreasing the expression of COX2.

Furthermore, upregulation of COX 2 expression may be a cause of cancer development, metastasis and chemoresistance (27). The present study revealed that inhibition of COX2 with CAY10404 enhanced the sensitivity of lung cancer cells toward sesamin by inducing apoptosis and G1-phase arrest. In addition, IL1 $\beta$, IL 6 and TNF $\alpha$ were confirmed as downstream molecules of COX2 (18). In the present study, cotreatment with CAY10404 and sesamin downregulated the levels of these molecules, indicating that COX2 is an important target of sesamin in lung cancer and that downregulated COX2 can improve the antitumor effect of sesamin. Li et al (28) observed that increased COX2 expression was associated with chemosensitivity and poor prognosis in cervical cancer, and that upregulated COX2 impeded chemosensitivity to dichloroacetate (DCA), while the combination of the COX2 inhibitor celecoxib with DCA enhanced the chemosensitivity to DCA in cervical cancer cells. Other studies have suggested that knocking down COX2 expression effectively increases the chemosensitivity of human gastric cancer cells (29) and laryngeal carcinoma cells (27). By contrast, a previous study indicated that $\mathrm{COX} 2$ is regulated by positive and negative mechanisms (30). Therefore, it is necessary to investigate the 

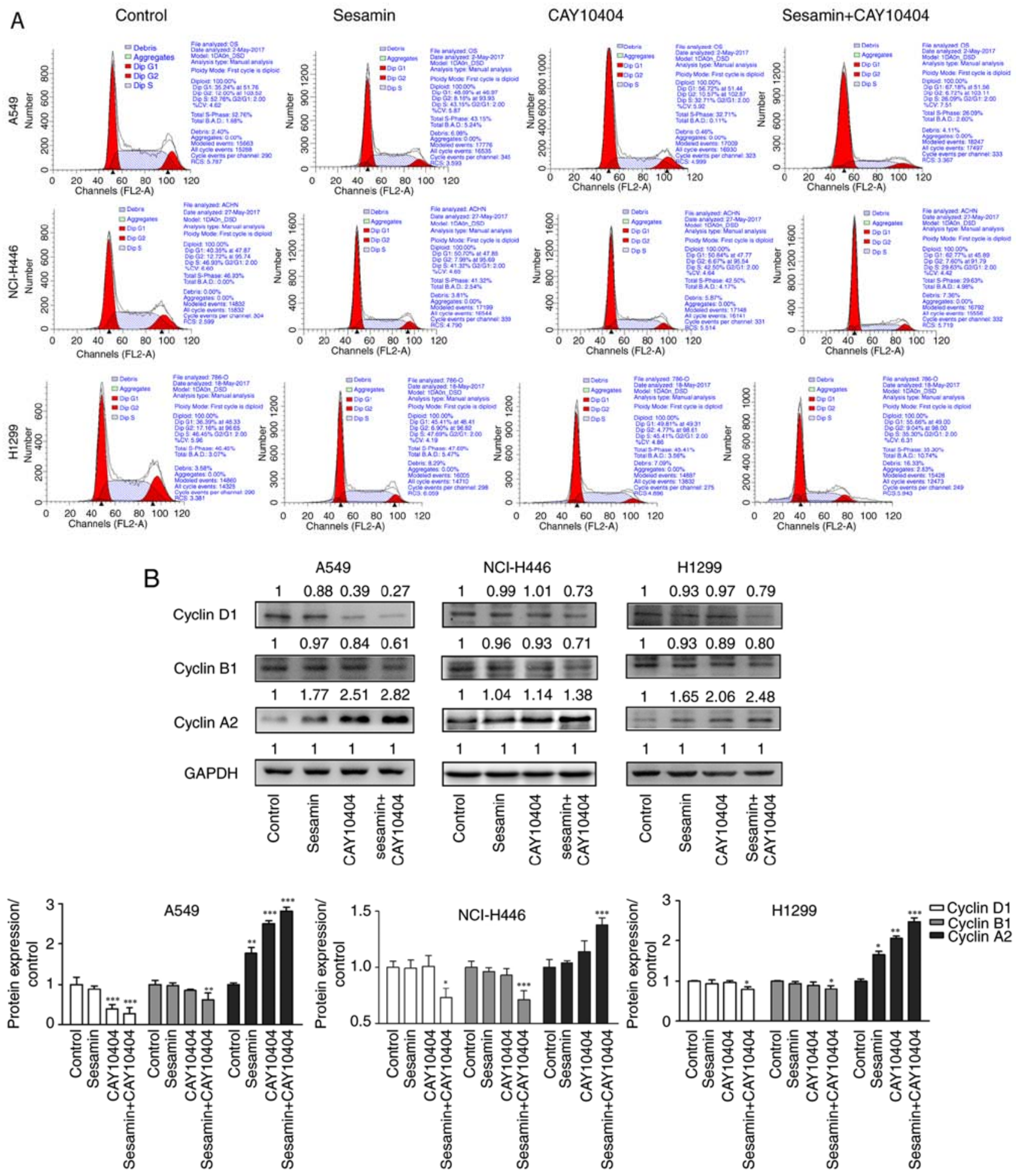

Figure 4. Cyclooxygenase inhibition enhances sesamin-induced cell cycle progression arrest at G1. (A) Lung cancer cells were treated with $25 \mu \mathrm{M}$ CAY10404 for $2 \mathrm{~h}$ and then incubated for additional $24 \mathrm{~h}$ with $50 \mu \mathrm{M}$ sesamin. Next, the cell cycle was assessed by flow cytometry upon staining with PI. (B) Cells were treated as in (A) and the expression of proteins associated with the cell cycle was evaluated using western blotting. ${ }^{*} \mathrm{P}<0.05,{ }^{* *} \mathrm{P}<0.01$ and ${ }^{* * * *} \mathrm{P}<0.001$ vs. the control.

role of the COX2 network in the sesamin-induced apoptosis of lung cancer cells to fully understand the oncogenic mechanisms of COX2.

A previous study reported that COX2 affects Akt activation, which is involved in bladder development (31). In the present study, the reduction in COX2 resulted in an increase in sesamin-induced Akt activity and a decrease in PI3K. In addition, the present results revealed that inhibition of PI3K with LY294002 improved apoptosis and induced cell cycle arrest at the G1 phase. Importantly, LY294002 decreased the expression of $\mathrm{PI} 3 \mathrm{~K}$, while partially upregulating the expression of COX2, although this effect was not statistically significant in A549 cells. These results indicated that sesamin inhibits the pAkt-PI3K signaling pathway by decreasing the expression 

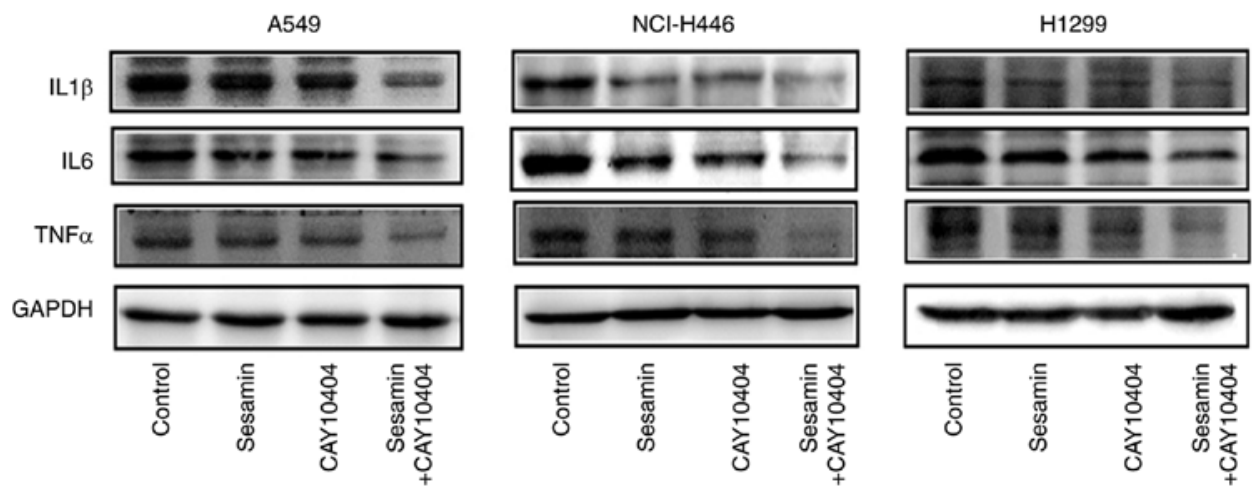

Figure 5. Combined treatment with COX2 and sesamin markedly reduces the expression of downstream molecules of COX2. A549, NCI-H446 and H1299 cells were treated with $25 \mu \mathrm{M}$ CAY10404 for $2 \mathrm{~h}$ and incubated for additional $24 \mathrm{~h}$ with $50 \mu \mathrm{M}$ sesamin. The expression of IL1 $\beta$, IL6 and TNF $\alpha$, which are downstream molecules of COX2, was assessed by western blotting. COX2, cyclooxygenase 2; IL, interleukin; TNF $\alpha$, tumor necrosis factor $\alpha$.

A

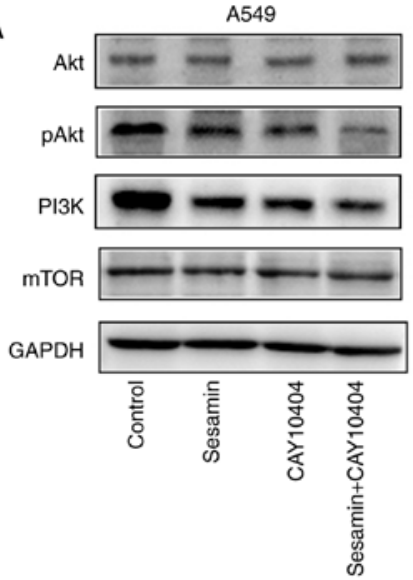

$\mathrm{NCl}-\mathrm{H} 446$

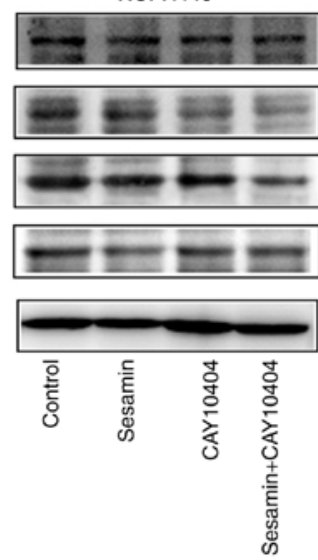

H1299

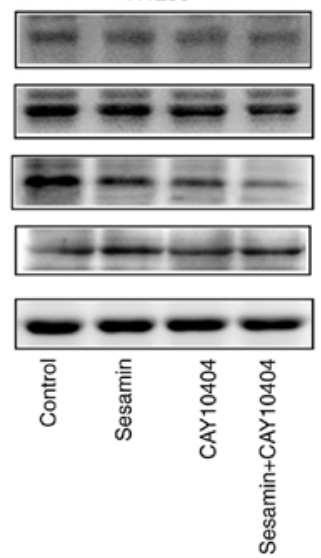

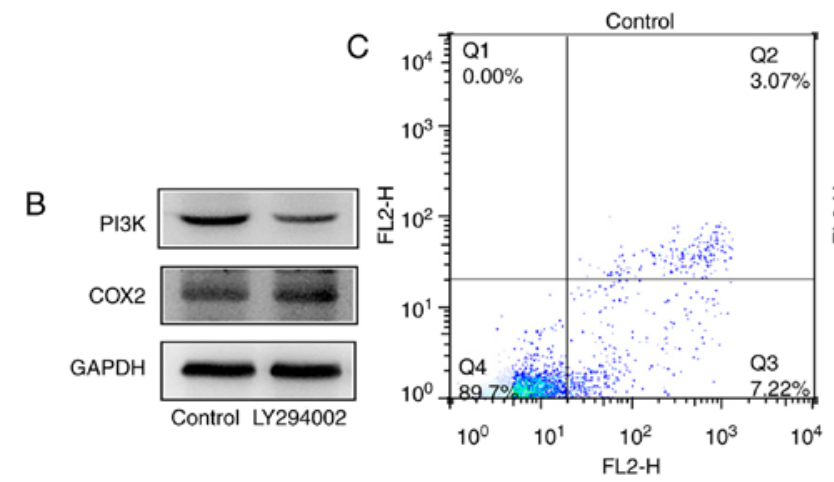

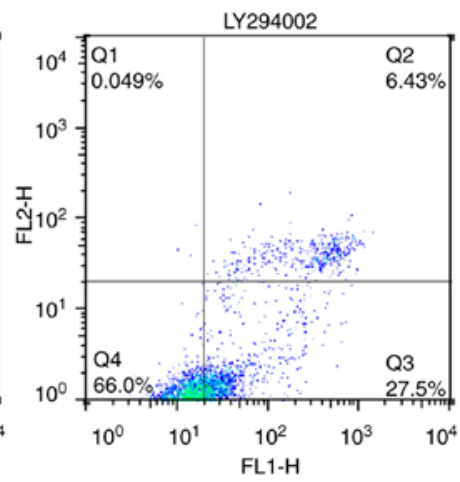

D

Control
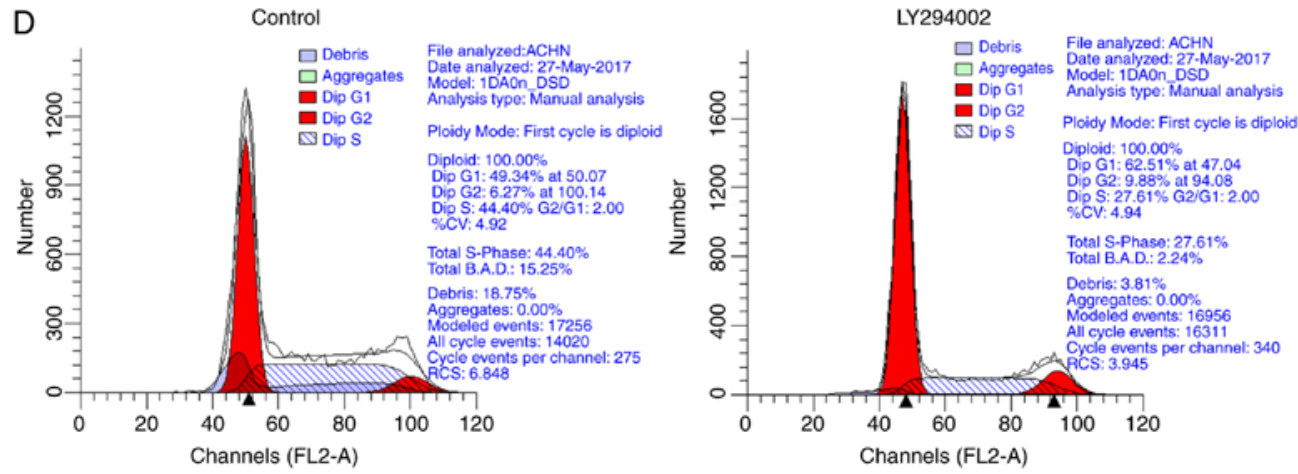

Figure 6. Decreased COX2 expression inhibits the pAkt-PI3K signaling pathway and mediates sesamin-induced apoptosis and cell cycle arrest in lung cancer cells. (A) Cells were treated with $25 \mu \mathrm{M}$ CAY 10404 for $2 \mathrm{~h}$ and incubated for an additional $24 \mathrm{~h}$ with $50 \mu \mathrm{M}$ sesamin. Next, the expression of Akt, pAkt, PI3K and mTOR was assessed by western blotting. (B) A549 cells were treated with $10 \mu \mathrm{M}$ PI3K inhibitor LY294002 for $24 \mathrm{~h}$ and the expression of PI3K and COX2 was assessed by western blotting. (C) A549 cells were treated as in (B) and cell apoptosis was analyzed by flow cytometry upon staining with Annexin V-fluorescein isothiocyanate/propidium iodide, while (D) the cell cycle was assessed by flow cytometry upon staining with PI. p-Akt, phosphorylated protein kinase B, PI3K, phosphoinositide 3 kinase; mTOR, mammalian target of rapamycin; COX2, cyclooxygenase 2. 
of COX2, therefore regulating cell cycle arrest and inducing apoptosis in vitro. In addition, COX2 and PI3K possibly form a negative feedback loop. However, further studies unraveling the detailed downstream effects of these molecules on cancer cells must be conducted.

In conclusion, the present study demonstrated that inhibition of COX2 expression enhanced the antitumor activity of sesamin via the Akt-PI3K signaling pathway in lung cancer cells. Therefore, a potential feedback loop comprising $\mathrm{COX} 2$ and $\mathrm{PI} 3 \mathrm{~K}$ that coordinates the apoptosis of lung cancer cells is proposed, which may pave the way for the development of potential treatment strategies for lung cancer using the combination of sesamin and a COX2 inhibitor.

\section{Acknowledgements}

Not applicable.

\section{Funding}

The present study was funded by the Natural Science Foundation of Ningbo (grant no. 2017A610245).

\section{Availability of data and materials}

The datasets supporting the conclusions of the present study are included within this article. The datasets used and/or analyzed during the current study are available from the corresponding author or the first author on reasonable request.

\section{Authors' contributions}

QW conceived and designed the study. QF wrote the manuscript. YZ was involved in revising manuscript critically for important intellectual content and given final approval of the version to be published. QF, MS, GG and $\mathrm{ZZ}$ conducted experiments, collected the data and performed statistical analysis. All authors read and approved the final version of the manuscript.

\section{Ethics approval and consent to participate}

Not applicable.

\section{Patient consent for publication}

Not applicable.

\section{Competing interests}

The authors declare that they have no competing interests.

\section{References}

1. Salskov A, Hawes SE, Stern JE, Feng Q, Jordan CD, Wiens L, Rasey J, Lu H, Kiviat NB and Vesselle H: Hypermethylation of CCND2 may reflect a smoking-induced precancerous change in the lung. J Oncol 2011: 950140, 2011.

2. Siegel RL, Miller KD and Jemal A: Cancer statistics, 2017. CA Cancer J Clin 67: 7-30, 2017.
3. Bao M, Song Y, Xia J, Li P, Liu Q and Wan Z: miR-1269 promotes cell survival and proliferation by targeting tp53 and caspase- 9 in lung cancer. Onco Targets Ther 11: 1721-1732, 2018.

4. Zheng L, Zhou H, Guo L, Xu X, Zhang S, Xu W and Mao W: Inhibition of NIPBL enhances the chemosensitivity of non-small-cell lung cancer cells via the DNA damage response and autophagy pathway. Onco Targets Ther 11: 1941-1948, 2018.

5. Thuy TD, Phan NN, Wang CY, Yu HG, Wang SY, Huang PL, Do YY and Lin YC: Novel therapeutic effects of sesamin on diabetes-induced cardiac dysfunction. Mol Med Rep 15: 2949-2956, 2017.

6. Fan D, Yang Z, Yuan Y, Wu QQ, Xu M, Jin YG and Tang QZ: Sesamin prevents apoptosis and inflammation after experimental myocardial infarction by JNK and NF- $\kappa \mathrm{B}$ pathways. Food Funct 8: 2875-2885, 2017.

7. Qiang L, Yuan J, Shouyin J, Yulin L, Libing J and Jian-An W: Sesamin attenuates lipopolysaccharide-induced acute lung injury by inhibition of TLR4 signaling pathways. Inflammation 39: 467-472, 2016.

8. Xu P, Cai F, Liu X and Guo L: Sesamin inhibits lipopolysaccharide-induced proliferation and invasion through the p38-MAPK and NF-kappaB signaling pathways in prostate cancer cells. Oncol Rep 33: 3117-3123, 2015.

9. Kuang W, Deng Q, Deng C, Li W, Shu S and Zhou M: Hepatocyte grow th factor induces breast cancer cell invasion via the PI3K/Akt and p38 MAPK signaling pathways to up-regulate the expression of COX2. Am J Transl Res 9: 3816-3826, 2017.

10. Chun KS and Surh YJ: Signal transduction pathways regulating cyclooxygenase-2 expression: Potential molecular targets for chemoprevention. Biochem Pharmacol 68: 1089-1100, 2004.

11. Spano JP, Chouahnia K and Morere JF: Cyclooxygenase 2 inhibitors and lung carcinoma. Bull Cancer 91 Spec No: S109-S112, 2004 (In French).

12. Yang CL, Zheng XL, Ye K, Ge H, Sun YN, Lu YF and Fan QX: MicroRNA-183 acts as a tumor suppressor in human non-small cell lung cancer by down-regulating MTA1. Cell Physiol Biochem 46: 93-106, 2018.

13. Yin H, Ma J, Chen L, Piao S, Zhang Y, Zhang S, Ma H, Li Y, Qu Y, Wang X and Xu Q: MiR-99a enhances the radiation sensitivity of non-small cell lung cancer by targeting mTOR. Cell Physiol Biochem 46: 471-481, 2018.

14. Jiang SX, Qi B, Yao WJ, Gu CW, Wei XF, Zhao Y, Liu YZ and Zhao BS: Berberine displays antitumor activity in esophageal cancer cells in vitro. World J Gastroenterol 23: 2511-2518, 2017.

15. Xiao H, Liu Y, Liang P, Wang B, Tan H, Zhang Y, Gao X and Gao J: TP53TG1 enhances cisplatin sensitivity of non-small cell lung cancer cells through regulating miR-18a/PTEN axis. Cell Biosci 8: 23, 2018.

16. Livak KJ and Schmittgen TD: Analysis of relative gene expression data using real-time quantitative PCR and the 2(-Delta Delta C(T) method. Methods 25: 402-408, 2001.

17. Zhuang H, Meng X, Li Y, Wang X, Huang S, Liu K, Hehir M, Fang R, Jiang L, Zhou JX, et al: Cyclic AMP responsive element-binding protein promotes renal cell carcinoma proliferation probably via the expression of spindle and kinetochore-associated protein 2. Oncotarget 7: 16325-16337, 2016.

18. Yoon KY, Kim KJ, Youn HS, Oh SR and Lee BY: Brazilin suppresses inflammation via the down-regulation of IRAK4 in LPS-stimulated Raw264.7 Macrophage. J Food Nutri Res 3: 575-580, 2015.

19. Alam SK, Astone M, Liu P, Hall SR, Coyle AM, Dankert EN, Hoffman DK, Zhang W, Kuang R, Roden AC, et al: DARPP-32 and t-DARPP promote non-small cell lung cancer growth through regulation of IKK $\alpha$-dependent cell migration. Commun Biol 1: 43, 2018.

20. Kong X, Ma MZ,Zhang Y, Weng MZ, Gong W, Guo LQ,Zhang JX, Wang GD, Su Q, Quan ZW and Yang JR: Differentiation therapy: Sesamin as an effective agent in targeting cancer stem-like side population cells of human gallbladder carcinoma. BMC Complement Altern Med 14: 254, 2014.

21. Akl MR, Ayoub NM and Sylvester PW: Mechanisms mediating the synergistic anticancer effects of combined $\gamma$-tocotrienol and sesamin treatment. Planta Med 78: 1731-1739, 2012.

22. Deng P, Wang C, Chen L, Wang C, Du Y, Yan X, Chen M, Yang $\mathrm{G}$ and $\mathrm{He} \mathrm{G}$ : Sesamin induces cell cycle arrest and apoptosis through the inhibition of signal transducer and activator of transcription 3 signalling in human hepatocellular carcinoma cell line HepG2. Biol Pharm Bull 36: 1540-1548, 2013. 
23. Harikumar KB, Sung B, Tharakan ST, Pandey MK, Joy B, Guha S, Krishnan S and Aggarwal BB: Sesamin manifests chemopreventive effects through the suppression of NF-kappa B-regulated cell survival, proliferation, invasion, and angiogenic gene products. Mol Cancer Res 8: 751-761, 2010.

24. Dhakal HP, Naume B, Synnestvedt M, Borgen E, Kaaresen R, Schlichting E, Wiedswang G, Bassarova A, Holm R, Giercksky KE and Nesland JM: Expression of cyclooxygenase-2 in invasive breast carcinomas and its prognostic impact. Histol Histopathol 27: 1315-1325, 2012.

25. Karavitis J, Hix LM, Shi YH, Schultz RF, Khazaie K and Zhang M: Regulation of COX2 expression in mouse mammary tumor cells controls bone metastasis and PGE2-induction of regulatory $\mathrm{T}$ cell migration. PLoS One 7: e46342, 2012.

26. Shimizu S, Fujii G, Takahashi M, Nakanishi R, Komiya M, Shimura M, Noma N, Onuma W, Terasaki M, Yano T and Mutoh M: Sesamol suppresses cyclooxygenase-2 transcriptional activity in colon cancer cells and modifies intestinal polyp development in Apc (Min/+) mice. J Clin Biochem Nutr 54 95-101, 2014.
27. Wang $\mathrm{R}$, Wang $\mathrm{X}$, Lin $\mathrm{F}$, Gao $\mathrm{P}$, Dong $\mathrm{K}$ and Zhang $\mathrm{HZ}$ : shRNA-targeted cyclooxygenase (COX)-2 inhibits proliferation, reduces invasion and enhances chemosensitivity in laryngeal carcinoma cells. Mol Cell Biochem 317: 179-188, 2008.

28. Li B, Li X, Xiong H, Zhou P, Ni Z, Yang T, Zhang Y, Zeng Y, He J, Yang F, et al: Inhibition of COX2 enhances the chemosensitivity of dichloroacetate in cervical cancer cells. Oncotarget 8: 51748-51757, 2017.

29. Chan MW, Wong CY, Cheng AS, Chan VY, Chan KK, To KF, Chan FK, Sung JJ and Leung WK: Targeted inhibition of COX-2 expression by RNA interference suppresses tumor growth and potentiates chemosensitivity to cisplatin in human gastric cancer cells. Oncol Rep 18: 1557-1562, 2007.

30. Liu S, Zhang C, Zhang K, Gao Y, Wang Z, Li X, Cheng G, Wang S, Xue X, Li W, et al: FOXP3 inhibits cancer stem cell self-renewal via transcriptional repression of COX2 in colorectal cancer cells. Oncotarget 8: 44694-44704, 2017.

31. Shimada K, Anai S, Marco DA, Fujimoto K and Konishi N: Cyclooxygenase 2-dependent and independent activation of Akt through casein kinase 2alpha contributes to human bladder cancer cell survival. BMC Urol 11: 8, 2011. 